\title{
Estrategias para enfrentar el Síndrome de Agotamiento Profesional: Aportes desde la institución y los trabajadores penitenciarios peruanos
}

\section{Strategies against Burnout Syndrome: Contributions from the institution and Peruvian penitentiary workers}

\author{
Lucia Bracco Bruce*, Raúl Valdez Oyague, Andrea Wakeham Nieri, Tesania Velázquez \\ Grupo de Investigación de Psicología Forense y Penitenciaria, Pontificia Universidad Católica del Perú, Lima, Perú \\ *lucia.bracco@pucp.pe
}

Recibido: 22-mayo-2018

Aceptado: 12-noviembre-2018

\section{RESUMEN}

El artículo presenta los resultados de una investigación cualitativa que tenía como objetivo analizar las estrategias para enfrentar el Síndrome de Agotamiento Profesional (SAP) y el estrés laboral en el contexto penitenciario peruano, desde la perspectiva de la institución y los trabajadores penitenciarios. Para ello, se desarrollaron 21 entrevistas semi-estructuradas a autoridades del Instituto Nacional Penitenciario (INPE) y 12 grupos focales donde participaron 59 trabajadores de las áreas de tratamiento y seguridad de 10 Establecimientos Penitenciarios (E.P.) a nivel nacional. A partir del discurso de los participantes se han construido las siguientes áreas de análisis: (a) Cambios estructurales en la institución, (b) Fortalecimiento de habilidades personales y profesionales y (c) Integración social del personal y entre las áreas. Frente a sus propuestas, se desarrolla un modelo sistémico que incluye las tres líneas de acción. Se enfatiza en la importancia de consolidar programas de intervención sistémicos y multidimensionales, reconociendo las opiniones de "abajo hacia arriba" que fomenten la participación de los trabajadores.

Palabras claves: estrategias, prevención, prisiones, síndrome de agotamiento profesional, trabajadores penitenciarios

\section{ABSTRACT}

The article presents the results of qualitative research that aimed to analyse the strategies to deal with Burnout Syndrome and work stress in the Peruvian penitentiary context. The research process supposed 21 semistructured interviews with authorities of the National Penitentiary Institute (INPE), and 12 focus groups with 59 workers from the treatment and security areas of 10 prisons nationwide. The paper describes three areas of analysis constructed throughout participants' discourses: (a) Structural changes in the institution, (b) Strengthening personal and professional skills and (c) Social integration of personnel and between areas. In that sense, this paper presents a systemic model to face Burnout Syndrome which includes an intertwined and dialogic program of the three mentioned areas. Moreover, it emphasises on the importance of consolidating systemic and multidimensional intervention programs and recognise "bottom-up" opinions which encourage worker participation.

Keywords: burnout syndrome, prevention, penitentiary staff, strategies

Financiamiento: Pontificia Universidad Católica del Perú, Lima, Perú.

Cómo citar este artículo: Bracco Bruce, L., Valdez Oyague, R., Wakeham Nieri, A., Velázquez, T. (2018). Estrategias para enfrentar el Síndrome de Agotamiento Profesional: Aportes desde la institución y los trabajadores penitenciarios peruanos. Psicoperspectivas, 17(3), 1-13. http://dx.doi.org/10.5027/psicoperspectivas-vol17-issue3-fulltext-1381

\begin{tabular}{|l|l}
\hline$(\boldsymbol{c c}) \mathrm{EY}_{\mathrm{EY}}$ \\
Publicado bajo licencia Creative Commons Attribution International 4.0
\end{tabular} 
El Síndrome de Agotamiento Profesional (SAP) consiste en un conjunto de procesos psicológicos que desarrollan los trabajadores de una institución como resultado del fracaso de las estrategias de afrontamiento utilizadas ante diversos estresores del entorno laboral (Ahola, ToppinenTanner, \& Seppanen 2017; Maslach, Schaufeli, \& Leiter, 2001; Maslach, 1993). Se presenta como consecuencia de la presencia constante de estrés laboral, definido como una serie de respuestas psico-fisiológicas a condiciones del ámbito laboral percibidas como amenazantes, que pueden ocasionar agotamiento siempre y cuando dichas condiciones se mantengan en el tiempo y el trabajador no pueda encontrar respuestas efectivas que permitan resolverlas (Navarro, López, Heliz, \& Real, 2018; Osorio \& Cárdenas, 2017). El SAP se presenta y evalúa en las dimensiones de cansancio emocional, despersonalización y baja realización personal (Shaufeli, Maslach, \& Marek, 1993). A partir de ello, se produce una reducción en el involucramiento de las tareas, desmotivación y disminución del sentido de eficacia. Como consecuencia, se deteriora la salud mental de los trabajadores y hay un distanciamiento del cumplimiento de los objetivos laborales (Breevart, Bakker, Hetland, \& Hetland, 2014; Demerouti, 2014; Leiter, \& Maslach, 2014).

Las investigaciones sobre el SAP han puesto énfasis en la prevalencia del constructo a través de su presentación sintomática y las causas personales y sociales que se relacionan (Aydemir, \& Icelli, 2014; Garcia, \& Herrero, 2010; Hemmeter, 2013; Maslach, 2003; MercadoSalgado, \& Gil-Monte, 2010; Velázquez et al, 2015). No obstante, aún falta profundizar sobre posibles estrategias de afrontamiento que permitan eliminar el malestar en los trabajadores (Leiter, \& Maslach, 2014), especialmente considerando los contextos laborales de manera específica, como en esta oportunidad, el contexto penitenciario peruano.

Diversos estudios con trabajadores penitenciarios encontraron que son una población vulnerable al SAP (García, \& Herrero, 2010; Rodríguez-Diaz, Rubin-Prieto, Bringas-Molleda, Alvarez-Fresno, \& Herrero-Olaizola, 2015). Por ejemplo, en un Establecimiento Pentenciario (E.P.) español, Hernández, Fernández, Ramos y Contador (2016) observaron que el $43.6 \%$ presenta prevalencia del SAP en grado alto. Por su parte, a partir de una investigación con guardias de seguridad en Colombia, Rodríguez (2014) concluye que, si bien no se encuentran índices altos de SAP, la mayoría de participantes tiene una dimensión comprometida, encontrándose en riesgo de padecerlo. Por otro lado, Rodríguez, Rubin, Bringas, Álvarez y Herrero (2015) encontraron que los trabajadores penitenciarios que emplean modelos de tratamiento alternativos, caracterizados por una mayor preocupación por alcanzar objetivos concretos de tratamiento, tienden a presentar menores índices de SAP.

Este artículo parte del reconocimiento que los trabajadores penitenciarios peruanos se desempeñan cotidianamente en condiciones laborales complicadas que producen altos índices de estrés laboral $y$, por consiguiente, un riesgo mayor de presentar SAP (Velázquez, et al., 2015). Características como sobrepoblación penitenciaria y hacinamiento en los Establecimientos Penitenciarios (E.P.) (Instituto Nacional Penitenciario [INPE], 2016), inadecuada infraestructura penitenciaria (INPE, 2016) y precariedad en la provisión de servicios básicos a los internos (salud, nutrición y educación) (Defensoría del Pueblo, 2013), ocasionan mayores dificultades a las complejas tareas que deben desempeñar los trabajadores penitenciarios. Lo descrito produce una excesiva carga laboral, y a las condiciones de precariedad, se suma el hecho que no se cuenta con suficiente personal penitenciario para cumplir adecuadamente los objetivos institucionales. Por ejemplo, actualmente, existen 68 E.P. a nivel nacional, con capacidad de albergue de 39156 presos, pero existen 88423 personas privadas de su libertad. Para responder a dicha demanda, la institución cuenta con 191 psicólogos, 155 asistentes sociales y 92 profesionales del área legal (INPE, 2018).

Tomando en cuenta lo anterior, a partir de una investigación a nivel nacional donde participaron 910 trabajadores de las áreas de tratamiento y seguridad, se encontró que $74 \%$ presentaba por lo menos un área comprometida (Bracco, Wakeham, Valdez, \& Velázquez, en prensa). A su vez, los trabajadores penitenciarios señalaron como causas del SAP: las precarias condiciones laborales que atentan contra su seguridad económica, legal, física y de salud mental; la debilidad institucional y poca transparencia en torno a la contratación, meritocracia, etc.; las conflictivas relaciones interpersonales; y la dificultad emocional que implica trabajar con personas privadas de su libertad (Bracco, Valdez, Wakeham, \& Velázquez, en prensa).

Este artículo da cuenta de uno de los resultados de la investigación nacional "Síndrome de Agotamiento Profesional y trabajadores penitenciarios Peruanos". En este artículo se nombran dos referencias que se encuentran "en prensa". La primera de ellas se titula "Síndrome de Agotamiento Profesional, Variables Sociodemográficas y Condiciones Laborales en Trabajadores Penitenciarios Peruanos" el cual da cuenta del análisis cuantitativo sobre la prevalencia del SAP y su correlación con variables socio demodráficas y laborales en 910 trabajadores penitenciarios a nivel nacional. La segunda referencia se refiere al artículo "Síndrome de Agotamiento Profesional y trabajadores penitenciarios peruanos. Una mirada cualitativa a los factores institucionales y sociales". La segunda referencia y el 
artículo que se presenta en esta revista desarrollan la información cualitativa de la investigación nacional. Esta información se levantó a través de entrevistas a actores claves y grupos focales a los trabajadores de las regiones de manera paralela al levantamiento de la data cuantitativa. Si bien en ambos artículos han analizado la información levantada con la misma muestra, se discuten puntos distintos. Por un lado, en uno se desarrollan los factores causales del SAP desde el punto de vista de los trabajadores. Por otro lado, en esta oportunidad se discuten las estrategias y acciones para enfrentar el síndrome desde la perspectiva de los trabajadores

Frente a esta realidad, urge proponer posibles acciones que puedan aportar a la construcción de bienestar de los trabajadores y al cumplimiento de los objetivos institucionales. De esta manera, el presente artículo tiene como objetivo identificar y analizar las estrategias que podrían implementarse para enfrentar el SAP, contribuyendo a la formulación de un modelo teórico fundado en la mirada de los trabajadores penitenciarios de distintas áreas de la institución. La relevancia teórica consiste en la contribución a la construcción de un modelo sistémico, compuesto de estrategias articuladas para integrar el trabajo entre distintas áreas de la institución en función a objetivos compartidos. Además, se plantea como alternativa ante los riesgos de efectuar intervenciones fragmentarias basadas en enfoques particulares que queden lejos del objetivo de responder a la complejidad del fenómeno del SAP. A nivel metodológico la perspectiva de los trabajadores muestra miradas variadas pero consistentes sobre la problemática del SAP que proveen puntos de partida sólidos para la puesta en marcha de futuras intervenciones (Bracco et al., en prensa). Para alcanzar los objetivos descritos anteriormente, se desarrolló una investigación cualitativa de alcance nacional entre el 2013 y el 2015, donde se realizaron entrevistas semiestructuradas a 21 autoridades del INPE y se desarrollaron 12 grupos focales con trabajadores de las áreas de seguridad y tratamiento de 10 E.P. del Perú.

Tomando en cuenta los avances teóricos, se describen las fortalezas y limitaciones de tres tipos de estrategias: estrategias centradas en la persona, estrategias centradas en la institución y estrategias mixtas.

\section{Estrategias centradas en la persona}

Estas estrategias identifican al individuo como el objetivo de la intervención (Leiter, \& Maslach, 2014; McCormack, \& Cotter, 2013). El SAP se manifiesta a través de distintos síntomas identificables de manera individual; por tanto, el propósito es transformar o fortalecer a la persona (Leiter, \& Maslach, 2014). Para ello se emplean técnicas sobre la base de la resiliencia (Leiter, \& Maslach, 2014), el fomento de la salud, la autoobservación (Karl, \& Fischer, 2013) y la atención en la manifestación del problema (Hurrel, 2005). En suma, se busca actuar frente a la manera cómo los individuos afrontan el estrés (Walter, Plaumann, \& Krugmann, 2013).

Las estrategias implican el desarrollo de una mejor salud física y mental, donde el autocuidado resulta fundamental. De acuerdo con Karl y Fischer (2013), el camino más adecuado para alcanzarlo es el desarrollo de una vida saludable, establecida por logros claros y concretos como suficiente sueño, actividad física regular, moderación en el consumo de alcohol, establecimiento de aficiones, relaciones interpersonales significativas y el empleo de técnicas de relajación. Alcanzar estos logros favorecerá la autoobservación, la que a su vez permitiría al trabajador ser consciente y detectar los comportamientos que influyen en la generación del SAP. Adicionalmente, Hemmeter (2013) destaca la importancia de fomentar competencias emocionales para reducir el estrés. De ser necesario, la persona puede contar con estrategias terapéuticas más específicas, concentradas principalmente en el fortalecimiento de la autoestima y la autoeficacia.

Entre los retos del presente modelo, Leiter y Maslach (2014) destacan que, si bien, a lo largo de los años la literatura se ha concentrado centralmente en este tipo de intervenciones, no hay evidencia suficiente o consistente sobre la efectividad de las mismas. Los autores sostienen que los estudios que dan cuenta sobre la eficacia de las mencionadas intervenciones son escasos y generalmente provistos de muestras pequeñas y planteadas a través de diseños de investigación por lo general débiles. Parte de la explicación de su mayor presencia se relaciona con que tienden a ser menos costosas para la institución, por lo menos a corto plazo. Además, existe una importante influencia teórica en tanto el SAP ha sido definido mayoritariamente en términos de experiencias individuales (cansancio emocional, despersonalización y realización personal).

\section{Estrategias centradas en la institución}

La perspectiva presentada en este acápite cuestiona la mirada individualista planteada en las estrategias centradas en la persona. La crítica supone que se concibe a los sujetos como responsables absolutos de sus desempeños y celebra el triunfo individual sobre cualquier obstáculo, reforzando la idea de que los estresores deben ser superados, en vez de eliminados. Por consiguiente, se opone al enfoque que localiza la fuente del SAP en el individuo definiéndolo como una posible persona débil, centrándose más en el espacio laboral (Leiter, \& Maslach, 2014).

De esta forma, las acciones comprendidas son el resultado 
de la identificación de aspectos institucionales que están asociados de forma importante con el nivel de satisfacción o malestar de los trabajadores y, por consiguiente, con la prevalencia del SAP. Dentro de la variedad de propuestas, los elementos tomados en cuenta que más destacan son el papel de las autoridades y los líderes (Kelloway, \& Dimoff, 2017; Hurrel, 2005), el nivel de confianza entre los miembros de la institución y de los miembros hacia la institución (Ballard, \& Grawitch, 2017); así como la circulación efectiva y transparente de información (Karl, \& Fischer, 2013).

Respecto a los líderes y su rol dentro de las instituciones, Kelloway y Dimoff (2017) consideran que un aspecto clave son las características y actitudes que tengan los mismos. Ellos son vistos como promotores de bienestar y facilitadores para el adecuado empleo de los recursos institucionales. En contraste, la presencia de líderes y supervisores con conductas hostiles y percibidas como arbitrarias han sido asociadas a baja autoeficacia y autoestima, así como a sentimientos de desesperanza. En consecuencia, se han implementado capacitaciones a líderes con el objetivo de que estén más pendientes de las necesidades del personal y que puedan canalizar de forma más adecuada los recursos. El punto más fuerte en el presente conjunto de estrategias consiste en que las variables identificadas han mostrado resultados favorables en el enfrentamiento del SAP.

En segundo lugar, la confianza y justicia organizacional son para Ballard y Grawitch (2017) factores críticos para establecer espacios psicológicamente saludables. Estas características se expresan cuando el personal percibe que las decisiones tomadas son justas, la información es compartida y el esfuerzo es reconocido.

En tercer lugar, la adecuada comunicación y circulación de información supone que puedan establecerse diálogos con facilidad, cooperar en todo momento y asistirse cuando sea necesario (McCormack, \& Cotter, 2013).

En suma, las intervenciones en el ámbito institucional resaltan por fomentar los niveles de participación, autonomía y poder de decisión dentro del espacio laboral (Walter, Plaumann, \& Krugman, 2013). Asimismo, para Leiter y Maslach (2014), la perspectiva institucional permite que los resultados alcanzados tengan más probabilidad de mantenerse en el tiempo. Ello se explicaría por el favorecimiento de la construcción de relaciones de soporte entre los colegas, principalmente promoviendo grupos de discusión acerca de la vida laboral. A pesar de que hay menores intentos de implementar y evaluar las estrategias centradas en la institución, los citados autores muestran que parecen tener un mayor impacto positivo frente al SAP.
El principal reto respecto a estas estrategias consiste en que las intervenciones realizadas en el nivel institucional han sido menos evaluadas, en comparación a las que emplean otros enfoques. Entre las principales razones destacan la alta complejidad de dichos programas, conectada con la dificultad de dar cuenta sobre sus resultados y efectos (Walter, Plaumann, \& Krugman, 2013).

\section{Estrategias mixtas}

Una tercera postura indica que el SAP debe ser considerado, tanto una dificultad del individuo como de la institución. Para Karl y Fischer (2013) los componentes individuales de la intervención buscan principalmente aumentar la resistencia al estrés, mientras que las acciones basadas en la institución se proponen mejorar las condiciones laborales minimizando los estresores externos y promoviendo el soporte social.

La orientación de estas intervenciones consiste en generar cambios en la organización laboral, promoviendo acciones de supervisión y consejería orientada a la construcción del trabajo en equipo. En la misma línea, Karl y Fisher (2013) hallaron que los grupos de supervisión que son moderados por psicoterapeutas e incluyen un consejero externo dirigidos a los trabajadores y sus superiores son el método más efectivo para afrontar el SAP. La evaluación de este tipo de programas muestra que los resultados tienden a producir cambios beneficiosos en el largo plazo, aunque se requiere de más estudios para precisar su efectividad (Walter, Plaumann, \& Krugman, 2013). Por su parte, Ahola, Toppinen-Tanner y Seppanen (2017) destacaron que en estudios revisados, en los que se utilizaban métodos combinados, los programas tenían en común involucrar a representantes laborales que pudieran promover la acción contra los aspectos detectados como causantes del SAP. Como resultado, pudo observarse una disminución del ausentismo y permisos por enfermedad en los seis meses siguientes.

\section{Método}

Para la investigación, se empleó una metodología cualitativa (Flick, 2018; Gaudet, \& Robert, 2018), con la cual se buscó ahondar en los discursos de los participantes a través de entrevistas semiestructuradas a autoridades del INPE; y grupos focales con trabajadores penitenciarios de las áreas de tratamiento y seguridad a nivel nacional. Se sostiene la importancia de reconocer y validar las percepciones de los trabajadores ya que suponen la base de la construcción de propuestas participativas, de "abajo hacia arriba", lo cual promueve el compromiso y la motivación por las transformaciones en el contexto laboral, con miras a la construcción de espacios más saludables a partir de sus propias recomendaciones (Ballard, \& 
Grawitch, 2017; Demerouti, 2014; Leiter, \& Maslach, 2014).

\section{Participantes}

Los participantes eran autoridades de la Sede Central del INPE y trabajadores de las áreas de seguridad y tratamiento de 10 E.P. del Perú. Los E.P. que fueron incluidos en la investigación se encuentran ubicados en las regiones de Lima, Centro Huancayo, Nor Oriente San Martín, Oriente Pucallpa, Sur Arequipa y Norte Chiclayo. Fueron elegidos a través del criterio de conveniencia, considerando: a) presencia de personal de seguridad y personal de tratamiento, b) representatividad de diferentes regiones en el país, c) E.P. más representativo de la región elegida y d) posibilidad de acceder a los E.P.

Los participantes fueron divididos en dos grupos. Por un lado, participaron 21 autoridades (16 hombres y 5 mujeres), que estaban ejerciendo cargos de poder en la Sede Central del INPE o en alguno de los E.P. que formaron parte de la investigación. Se tomó en consideración como criterio de inclusión que se encuentren desempeñando dicho cargo directivo al momento de la investigación. Se incorporó la mirada de las autoridades con la finalidad de analizar el enfoque institucional sobre las acciones para enfrentar el estrés laboral y el SAP en el sistema penitenciario. Las autoridades son responsables de la gestión de equipos y tomadores de decisiones, desempeñándose como directores de seguridad, directores de tratamiento, jefes de personal o coordinadores de área.

Por otro lado, participaron trabajadores de las áreas de seguridad y tratamiento de los E.P. a nivel nacional. Se organizaron 12 grupos focales: 7 fueron con trabajadores del área de tratamiento, donde participaron 35 personas (23 hombres y 12 mujeres); y 5 fueron con trabajadores del área de seguridad donde participaron de 24 personas (18 hombres y 6 mujeres). Como criterios de inclusión, se tomó en cuenta que estén trabajando en ese momento en uno de los E.P. Al conformar cada grupo se prestó atención a que al interior de los mismos estuvieran representadas las diferentes sub áreas de cada una de estas dos grandes unidades. Por consiguiente, en los grupos focales del área de seguridad, hubo profesionales encargados de la seguridad externa e interna de cada E.P. En los grupos focales con el personal del área de tratamiento estuvieron presentes representantes de áreas como educación, salud, psicología, trabajo social y legal.

Para la selección de participantes se llevó a cabo un muestreo por conveniencia (no probabilístico), tomando en cuenta la disponibilidad de la persona y su pertenencia a algunos de los dos grupos de interés.
Técnicas de recolección de información

Se emplearon dos medios para la recolección de información: la entrevista semiestructurada y el grupo focal.

La entrevista semiestructurada registra de forma abierta el discurso del participante, que incluye sus percepciones, creencias y afectos sobre diferentes temas que se propongan (Hennink, Hutter, \& Bailey, 2011). En este caso, se llevó a cabo 3 entrevistas a autoridades de la Sede Central de INPE y 18 entrevistas a autoridades de los E.P.

Respecto a los grupos focales, la técnica supone recolectar información a partir de proponer una temática a los participantes. Se diseñaron preguntas que buscaban profundizar en el estrés laboral y en el SAP, con la finalidad de recoger los sentimientos, percepciones, experiencias y reacciones en los participantes (Beck, Bryman, \& Futing, 2004). Con los grupos focales se esperó contar con un número de participantes amplio, que brinde una mirada de las acciones laborales de los trabajadores penitenciarios y cómo vivencian y enfrentan el estrés y el SAP de manera cotidiana. Se dividió los grupos en dos áreas tomando en cuenta la organización institucional, la cual también está dividida en las áreas de seguridad y tratamiento. Como se ha mencionado, fueron realizados 12 grupos focales, de los cuales 7 fueron integrados por trabajadores del área de tratamiento y 5 estuvieron conformados por trabajadores del área de seguridad.

Las entrevistas semiestructuradas y los grupos focales se realizaron teniendo en consideración tres temas orientadores que fueron definidas a partir de la investigación sobre SAP en trabajadores penitenciarios realizada en dos E.P. de Lima, Perú (Velázquez, et al., 2015). Los temas son:

Causas de estrés laboral y SAP en el contexto penitenciario: se discutió sobre los posibles factores asociados al estrés laboral y al SAP, especialmente en los factores institucionales y relacionales. Además, se invitó a los participantes a dar ejemplos de sus respuestas.

Consecuencias del estrés laboral y SAP en los trabajadores: se buscó profundizar en las maneras cómo se manifiesta el estrés laboral y el SAP en las personas y las relaciones sociales e institucionales. Se preguntó de manera específica sobre las consecuencias físicas y relacionales (con las autoridades, pares laborales y con los internos e internas). Nuevamente, se solicitó a los participantes algunos ejemplos.

Estrategias para afrontar el estrés laboral y SAP: tuvo como finalidad preguntar acerca de las estrategias que recomendarían para enfrentar el estrés laboral y el SAP, así 
como identificar acciones que ya se vienen realizando.

\section{Procedimiento}

Se conformó un equipo de investigación interinstitucional integrado por miembros de las dos instituciones encargadas de la investigación. En este contexto de cooperación, se eligieron los E.P., se solicitaron los permisos a la Sede Central y directores de cada E.P. Las entrevistas semiestructuradas y los grupos focales se realizaron, según fuera el caso, en las oficinas de las autoridades correspondientes o en los mismos E.P. donde laboran los participantes, con la finalidad de garantizar la comodidad y privacidad de los mismos. Al final de cada entrevista y grupo focal se entregó material informativo sobre el SAP.

Para asegurar el cumplimiento de estándares éticos, todos los participantes fueron convocados de forma voluntaria a ser parte del estudio. Antes del inicio de la investigación se revisó el consentimiento informado donde se señalaban los objetivos y el proceso de investigación. Finalmente, para asegurar la confidencialidad y anonimato de los participantes, en este artículo se evita la mención de nombres, cargos o datos que permitan identificar a los participantes o a los E.P.

Concluida la etapa de recolección de información, los audios fueron transcritos para ser analizados en detalle a través del método de análisis temático (Braun, \& Clarke, 2006). Este análisis permite identificar patrones contenidos en un grupo particular de datos. En ese sentido, se construyeron núcleos de sentidos que dan la posibilidad de organizar, analizar y reportar temas recurrentes o patrones (Braun, \& Clarke, 2006; Gomes, 2012). Inicialmente, tres investigadores leyeron todo el material transcrito. Luego, se organizó la información considerando las áreas de la investigación (causas, consecuencias y estrategias frente al SAP). La lectura inicial aporta a que los investigadores se familiaricen con los discursos y se realice una primera codificación abierta. Finalmente, se propusieron áreas de análisis, la construcción de categorías para cada uno de ellas y las relaciones entre las mismas (Muñoz, 2003). Las categorías no fueron preestablecidas, sino que se construyeron a partir del discurso de los participantes. Para el análisis temático se usó como soporte el programa ATLAS-TI.

\section{Resultados}

Este artículo se centra en las estrategias que proponen los participantes, las cuales han sido construidas a partir de los factores sociales e institucionales que han identificado como causantes del estrés laboral y SAP (Bracco, et al., en prensa). Frente a ello, se observa que las causas del SAP muestran diversas áreas en conflicto $y$, por tanto, para afrontarlo se debe construir una mirada sistémica, donde se aborden las diferentes aristas propuestas por los trabajadores. De esta manera, se ha construido tres áreas de análisis, las cuales agrupan las categorías narradas por los participantes en: (a) Cambios estructurales en la institución, (b) Fortalecimiento de habilidades personales y profesionales, y (c) Integración social del personal y entre las áreas.

Las tres áreas construidas responden a una integración de los tres tipos de estrategias presentadas en el Marco de Referencia del presente artículo, las cuales han sido abordadas teóricamente desde posicionamientos epistemológicos distintos sobre cómo se debe afrontar el SAP. No obstante, se ha buscado dar cuenta de las percepciones de los trabajadores y la comprensión de un clima laboral complejo -que se ve atravesado por condiciones laborales precarias- $y$, por tanto, implica reconocer una multiplicidad de dimensiones que deben ser tomadas en cuenta con miras a la eliminación de esta problemática. De esta manera, más que excluyentes o contradictorias, se considera que las áreas construidas a partir de las propuestas de los participantes aportan a la construcción de una mirada sistémica de su espacio laboral, donde en efecto, estas estrategias deben ser puestas en acción -con mayor y menor urgencia- para construir espacios de bienestar y psicológicamente saludables.

Finalmente, para la construcción de las áreas y las categorías, se han considerado dos puntos importantes. En primer lugar, los temas abordados en las entrevistas semiestructuradas y los grupos focales estuvieron centradas en las causas, consecuencias y acciones. Si bien en este artículo hay un mayor análisis del tercero, todos dieron información valiosa para la construcción de categorías. En segundo lugar, se ha detallado que los participantes fueron divididos en dos grupos. No obstante, para el análisis se ha integrado los discursos ya que no se encuentran brechas discursivas entre los mismos. Esto puede ser debido a la constante rotación de los trabajadores de la institución. Debido a políticas institucionales del INPE, las personas asumen cargos administrativos, de autoridad institucional y de trabajadores penitenciarios de seguridad y/o tratamiento dentro de los E.P., los cuales van cambiando de manera constante y ocupando puestos diversos a lo largo de su carrera institucional, sin que haya necesariamente una línea de carrera ascendente como se concibe en otras instituciones.

\section{Cambios estructurales en la institución}

En esta área se incluyen las categorías que buscan incidir en la gestión organizacional laboral, lo que implica cambios estructurales que requerirían de creación de políticas 
institucionales y una mayor inversión presupuestal. Aparecen las siguientes categorías: a. Espacio y logística y b. Gestión de recursos humanos y c. Seguridad en el trabajo.

La categoría espacio y logística da cuenta de la importancia de contar con infraestructura, materiales y equipos de trabajo mínimos para cumplir su trabajo de manera eficiente $y$ tener un entorno que posibilite fortalecer el bienestar laboral. Los participantes refieren que los espacios donde desarrollan sus actividades laborales (reuniones de trabajo, llenado de informes, terapias, talleres individuales o grupales con internos e internas, etc.) deben ser reestructurados. En la mayoría de E.P. el número de internos excede la capacidad de albergue (INPE, 2016), por tanto, los niveles de hacinamiento representan una dificultad no sólo para la vivencia digna de los internos e internas, sino también para que los trabajadores penitenciarios puedan desempeñar efectivamente sus tareas cotidianas. Por ejemplo, un participante señala:

'Las condiciones institucionales, en cuanto a infraestructura, los espacios son pequeños. Cuánto quisiéramos que nos den espacios cómodos, pero también nosotros tenemos que adaptarnos a la infraestructura que el Estado nos da.' (Participante área de Tratamiento)

Sumado a ello, los participantes explicitan que requieren de espacios adecuados para descansar. Así, señalan que la mayoría de E.P. no cuenta con lugares donde el personal pueda almorzar, alejado de la población penitenciaria o descansar entre las diferentes actividades de su jornada diaria.

En la misma línea, en relación a la logística, los participantes mencionan la importancia de contar con recursos materiales y equipos que les permitan organizarse mejor y de manera más eficiente. Si bien el personal penitenciario refiere que hay necesidades compartidas (computadoras y materiales de escritorios), hay necesidades específicas por área. En el caso del personal de seguridad, recomiendan que se pueda adquirir implementos que faciliten el desempeño laboral cotidiano (lapiceros, cuadernos para poder elaborar los informes diarios de incidencia dentro del E.P.), así como, equipos para situaciones específicas del contexto penitenciario como la toma de rehenes, un motín o los traslados de internos a otros E.P. En el caso del área de tratamiento, los participantes también refieren que necesitan contar con materiales de escritorio, así como con herramientas actualizadas para cada área de trabajo (fichas de datos, materiales para realizar talleres de tratamiento y/o pruebas psicológicas, entre otras).
La segunda categoría de este eje, gestión de recursos humanos, da cuenta de cómo los participantes refieren al fortalecimiento y elaboración de políticas institucionales acerca de la contratación, ascensos, rotaciones y sanciones. Tanto en el tema de contratación de nuevo personal como ascensos dentro de la institución, los participantes solicitan que prime la meritocracia donde se considere el desempeño y competencias profesionales; así como, los estudios académicos. Desde la percepción de los participantes, el fortalecimiento de la meritocracia y la transparencia de dichos procesos ayudarían a consolidar la lucha anticorrupción que viene impulsando la institución y propiciaría que el personal se sienta reconocido:

\begin{abstract}
'Debe haber una meritocracia. Hay mucho personal que tiene la experiencia y la capacidad para poder dirigir los penales, pero por cuestiones políticas se eligen a dedo. En cierto modo, el personal no se siente reconocido por sus esfuerzos o por sus capacitaciones que haya podido realizar.' (Participante área de Seguridad)
\end{abstract}

Finalmente, la categoría seguridad en el trabajo, supone construir normas claras de cuidado para los trabajadores penitenciarios, particularmente en los temas de salud física, salud mental y apoyo legal.

Los participantes señalan que para sentirse seguros en el contexto penitenciario la institución debería proveer protocolos claros sobre cómo trabajar con internos e internas, en caso de que tengan enfermedades infecciosas (TBC, VIH, entre otras) o en situaciones de insalubridad como es el caso de muchos E.P.- por motivos de hacinamiento y ausencia de recursos. Asimismo, sostienen la importancia de contar con protocolos para casos de emergencia como incendios o terremotos, y de manera más específica para el contexto penitenciario, para casos de motines o rehenes. Además, que dichos protocolos se realicen de manera paralela a talleres de capacitación sobre cómo prevenir y/o manejar esas situaciones.

Sumado a lo anterior y en la línea de contar con mayor seguridad en la salud física, los participantes plantean que la institución debería contar con convenios para una adecuada atención médica y tratamiento por enfermedades, que puedan ser adquiridas realizando el trabajo penitenciario:

'Con respecto a las enfermedades, es necesario que la institución nos haga convenio con alguna clínica para que pueda, permanentemente, evaluar al personal que trabaja con personas que vienen de diferentes lugares y con diferentes enfermedades.' (Participante área de Tratamiento)

En la misma línea, el personal reconoce la necesidad de contar con atención psicológica, a nivel individual o grupal, 
para fortalecer su salud mental y contrarrestar el estrés que genera trabajar con personas que han delinquido y en entornos que consideran pueden ser violentos $\mathrm{y} / \mathrm{o}$ desprotegidos.

Finalmente, y de manera muy reiterada, los participantes señalan la importancia de contar con seguridad legal. Sostienen que en ocasiones el trabajador penitenciario se ve expuesto a denuncias por parte de los internos e internas por presuntas faltas dentro de su desempeño laboral. Frente a ello, los participantes refieren que hay instituciones como la Defensoría del Pueblo -organismo constitucionalmente autónomo encargado de velar por la buena administración pública en favor de los ciudadanos(Defensoría del Pueblo, 2017) que protege a los internos, pero que esto no se cumple en el caso del trabajador penitenciario. Por ejemplo, la siguiente cita describe la situación: "Yo creo que con una defensoría del agente penitenciario. Que haya abogados." (Participante área de Seguridad)

\section{Integración social del personal y entre las áreas}

Esta área busca trabajar en la construcción de vínculos y redes de soporte entre los trabajadores de la institución. Las dos categorías de esta área son: a. Integración entre trabajadores y b. Diálogo y coordinación entre áreas. Ambas tienen como objetivo mejorar las relaciones interpersonales entre los trabajadores. Se espera que las personas puedan ser más empáticas y respetuosas entre sí y, por tanto, poder construir un clima de colaboración entre las áreas que impacte positivamente en el clima laboral.

Por un lado, los participantes sostienen de manera reiterada que se puede impulsar la integración entre trabajadores a través de actividades recreativas. En muchos E.P. son los mismos trabajadores quienes las organizan, las cuales son acciones autogestionadas, que van desde actividades muy puntuales como la celebración de cumpleaños, hasta actividades que requieren de una organización más compleja como campeonatos deportivos inter-áreas. Los participantes las definen como actividades que fortalecen los vínculos de confianza y que, en la mayoría de casos no están institucionalizadas, sino que dependen de la motivación y organización de las autoridades y los trabajadores de cada E.P.

En la misma línea, los participantes recomiendan de manera reiterada que la institución promueva espacios de confraternización fuera de los E.P. como actividades lúdicas y deportivas. Los participantes mencionan que la finalidad es disminuir el estrés a través de la construcción de redes de confianza entre los trabajadores y el fortalecimiento de la "la identidad como institución". Es decir, se resaltan la importancia de la construcción de una identidad grupal laboral, que ofrezca identificación y empatía grupal, como medida para enfrentar el estrés laboral y el SAP.

De manera menos frecuente, los participantes también mencionan la necesidad de contar con reuniones, dentro del horario de trabajo, para compartir las dificultades laborales y las emociones que se generan al trabajar con los internos e internas. Los participantes mencionan que éstas buscan fortalecer las redes de soporte social y mejorar la comunicación entre pares.

Por otro lado, la categoría diálogo y coordinación entre áreas, da cuenta de cómo todos los participantes de los E.P. y las autoridades de la Sede Central del INPE, mencionan la necesidad de "alinear" los objetivos institucionales entre las áreas. De esta manera, identifican que se deben organizar reuniones de trabajo para establecer objetivos comunes y fortalecer acciones cooperativas entre el personal de las áreas de seguridad y de tratamiento. Los participantes mencionan, que si bien, la misión institucional se centra en la reinserción a la sociedad de las personas privadas de libertad (INPE, 2017), en la práctica se establece un quiebre entre los objetivos y funciones del personal del área de seguridad y del área de tratamiento.

Por un lado, el personal del área de seguridad se enfoca en el control y vigilancia de los E.P., mientras que el personal del área de tratamiento, estaría enfocado en los procesos y tareas que posibilitan la reinserción y reeducación de las personas privadas de libertad. Si bien, los participantes señalan que son conscientes que cada área cumple tareas complementarias dentro del sistema penitenciario, se dificulta el trabajo de manera cooperativa en la cotidianeidad laboral. Como una manera de solucionar esta problemática, los participantes refieren que se deben impulsar, de manera institucional, reuniones donde se pueda esclarecer los objetivos, roles y funciones de cada una de las áreas, así como, determinar cuáles serían las acciones de cooperación entre ambas.

'(...) hay que trabajar en eso para unificar, para coordinar entrelazar, hacerles entender al personal que un área depende de la otra área, seguridad depende de tratamiento, administración. Todos necesitamos, tenemos que trabajar coordinadamente. Si no existen esas coordinaciones, ese enlace, no se va a trabajar bien. Y aquí sí, también, el cansancio, los problemas que se han visto de cómo se presenta el agotamiento, se va a producir.' (Participante Autoridad)

En resumen, ambas categorías implican fortalecer vínculos de confianza y redes sociales entre el personal, pero con la finalidad de construir una institución donde prima el trabajo colaborativo. En ese sentido, para mejorar el trabajo en equipo y el fortalecimiento de la productividad, 
no basta con explicitar los objetivos institucionales de manera informativa, sino que supone un trabajo en la dimensión afectiva. De esta manera, se debe fortalecer habilidades sociales como trabajo en equipo, respeto, asertividad enfocada en cuáles son las tareas que deben cumplir dentro del espacio laboral.

\section{Fortalecimiento de habilidades personales y profesionales}

En esta área se incluyen las acciones que buscan fortalecer las capacidades personales, (mayormente psicológicas) y profesionales de los trabajadores penitenciarios. Se identifican dos categorías: a. Habilidades personales y b. Habilidades profesionales.

En relación a las habilidades personales, los participantes recomiendan que la institución debe fomentar talleres psicológicos para aprender a manejar el estrés laboral y prevenir el SAP. De manera específica, señalan que los talleres tendrían que trabajar en técnicas de relajación y motivación de los trabajadores. Algunos participantes plantean la posibilidad de hacerlo a través de técnicas artísticas como danza o arte. Uno de ellos indica:

'(...) podría ser... lo que es cuestión de capacitación o tratamiento personal, tratamiento psicológico, talleres psicológicos para poder mejorar el estrés, controlar.' (Participante Autoridad)

Sobre la categoría habilidades profesionales, los participantes refieren que sería fundamental recibir capacitaciones sobre la realidad penitenciaria. Hay un deseo de contar con actualizaciones constantes sobre nuevos conocimientos normativos, académicos y tecnológicos que les permitan mejorar su rendimiento profesional y comprender mejor el contexto penitenciario. Si bien, se menciona que desde la Sede Central del INPE hay un esfuerzo por impulsar capacitaciones, los participantes indican que las convocatorias y/o la difusión de las mismas pueden mejorarse. Asimismo, a diferencia de las acciones para fortalecer las habilidades sociales e integración, las cuales eran mayoritariamente impulsadas por los trabajadores, los participantes mencionan que las capacitaciones para fortalecer las habilidades profesionales no pueden ser impulsadas por las autoridades de cada E.P. ni por ellos mismos, sino que deben ser promovidas por la Sede Central. Como menciona uno de los participantes:

'Nosotros necesitamos capacitarnos en las normas, disposiciones sobre el tratamiento, modificaciones constantes que hay de las normas legales, eso. Porque nosotros trabajamos en contacto directo con los internos y la versión de quien tiene que salir la verdad es de nosotros. Nosotros, para poder llevar a cabo necesitamos capacitarnos. Estamos trabajando programas de tratamiento, pero que se están implementando, porque Lima manda la aplicación de estos programas pero no nos dan la adecuada capacitación.' (Participante área de Tratamiento)

En resumen, ambas categorías están centradas en las personas. Si bien se menciona la importancia del fortalecimiento de las habilidades personales, ambas categorías implican contar con mejores herramientas para desempeñarse en el espacio laboral. De esta manera, la categoría habilidades personales implica contar con mayores recursos emocionales para afrontar la dura y compleja realidad penitenciaria. Por su lado, la categoría habilidades profesionales implica fortalecer las capacidades académicas de los trabajadores en temas relacionados a su desempeño laboral, construyendo herramientas que les permitan comprender y relacionarse mejor en el contexto penitenciario. A partir de estas, los trabajadores pueden sentir mayor confianza en su desempeño y contrarrestar los efectos del estrés laboral y SAP.

\section{Discusión y conclusiones}

A partir de los resultados expuestos, se observa que los participantes visibilizan dificultades a diferentes niveles y presentan alternativas que, si se llevaran de manera conjunta, podrían enfrentar de manera sistémica la problemática del SAP. Entonces, tomando en cuenta las áreas y categorías identificadas en los resultados se ha elaborado una propuesta de modelo de intervención. Se trata de un modelo que requiere una acción conjunta a diferente nivel, donde los cambios estructurales en la institución dialoguen con la integración social del personal y entre las áreas, así como con el fortalecimiento de capacidades personales y profesionales. A continuación, se detalla cada uno de los elementos del modelo propuesto.

\section{Cambios estructurales en la institución}

Los participantes resaltan la importancia de crear cambios estructurales a nivel de infraestructura, adquisición de recursos logísticos y elaboración de políticas institucionales que impulsen transparencia en la gestión de recursos humanos y una sensación de protección -simbólica y concreta- a partir de políticas de seguridad y salud en el trabajo. Son las variables políticas y económicas hacinamiento, insuficiencia de presupuesto e informalidad y corrupción- que están arraigadas de manera estructural, lo que incide directamente en la prevalencia del SAP de los trabajadores penitenciarios (Bracco, et al., en prensa).

Es importante señalar que la ausencia de espacios y recursos logísticos no es una dificultad que sólo se encuentra en el sistema penitenciario. En diversas investigaciones sobre SAP en funcionarios públicos en el Perú, se han encontrado las mismas demandas. En ese 
sentido, en investigaciones con profesionales de la salud (Cáceres, 2013; Santivañez, 2016) y con profesionales de la salud mental (Yepez, 2015) se observa nuevamente la excesiva carga laboral por la sobrepoblación y la ausencia de espacios y recursos materiales para hacerle frente. De esta manera, es una problemática que se reproduce de manera estructural en el Perú y que nos evidencia cómo la precariedad institucional tiene un impacto en la salud mental del personal.

A pesar de ello, como señala Gil-Monte (2005), estas medidas suelen ser escasas ya que involucran inversiones presupuestales considerables. Así, aunque la sintomatología del SAP se manifiesta de manera individual, las investigaciones demuestran que son los factores institucionales los que juegan un rol protagónico en la aparición de los mismos. En ese sentido, se debe profundizar en la construcción de espacios de trabajo que tomen en cuenta transformaciones estructurales que apunten al bienestar de sus trabajadores (Ballard, \& Grawitch, 2017), lo cual incluye contar con espacios concretos para trabajar de manera segura, implementos logísticos y herramientas de trabajo, convenios legales, de salud y de salud mental con instituciones que puedan atender a los trabajadores y aumentar la contratación de personas. No obstante, también se debe trabajar en los sistemas de informalidad y corrupción (Bracco, et al., en prensa). Tal como plantea Maslach (2003), la ausencia de justicia en el espacio laboral incide directamente en la presencia del SAP. Ante ello, sería necesario consolidar y difundir protocolos transparentes de la gestión de recursos humanos de la institución.

\section{Integración social del personal y entre las áreas}

En este punto cabe resaltar la importancia que otorgan los participantes a las relaciones interpersonales y a la construcción de redes de soporte dentro del espacio institucional, habiendo sido definidas como una de las principales razones de prevalencia de SAP en la institución (Bracco, et al., en prensa). Los participantes proponen acciones para mejorar la calidad de las relaciones interpersonales, las cuales son actividades grupales con diversas finalidades: de recreación, espacios para compartir dificultades y emociones en relación con el trabajo y espacios de coordinación entre áreas.

En relación a las acciones de recreación y considerando la categoría integración entre áreas, como señala Demerouti (2014), resulta interesante reconocer las acciones que eligen impulsar los individuos dentro de sus espacios laborales para enfrentar las situaciones de estrés. Como sostiene el autor, en contextos de recursos disminuidos, como es el ámbito penitenciario, es difícil mantener de manera sostenida el mismo tiempo y energía para cumplir con los objetivos institucionales de manera óptima. Así, los trabajadores también encuentran estrategias como el humor, la celebración, el distanciamiento psicológico, la búsqueda de espacios de relajación y esparcimiento -como los campeonatos deportivos autogestionados por los trabajadores penitenciarios- para poder sobrellevar la vida cotidiana laboral. Demerouti (2014) señala que las acciones voluntarias por parte de los trabajadores no deben ser categorizadas como actividades únicamente recreativas, sino como acciones que, con poca movilización de recursos, fortalece la motivación y las relaciones de soporte social entre trabajadores.

En segundo lugar, los participantes también proponen realizar talleres para compartir las emociones que emergen al trabajar en el contexto penitenciario. Si bien, esta no es una recomendación mayoritariamente señalada por los participantes, siguiendo a McCormack y Cotter (2013), se quiere resaltar que esta estrategia promueve el autocuidado y el fortalecimiento de redes de soporte social dentro del espacio laboral considerando que, por lo general, las personas que padecen síntomas de estrés laboral o SAP, tienen dificultades para reconocerlos. Esto será posible, en la medida, que se impulse un diálogo entre los trabajadores, caracterizado por la expresión de los sentimientos y la aspiración a encontrar una retroalimentación e identificación entre los mismos. Estas reuniones de soporte se convierten en un ejercicio de autoconciencia con efectos catárticos, de fortalecimiento de redes de soporte social y de desarrollo de nuevas acciones impulsadas por los trabajadores que puedan tener efectos positivos frente al SAP.

De la misma forma, Olabarria y Mansilla (2007) sostienen que puede presentarse mayor prevalencia de SAP en personas que se desempeñan en tareas de servicio donde hay mayor contacto con daño y violencia, como es el caso del personal que trabaja en el sistema penitenciario. Para los autores, las personas que trabajan dentro de espacios considerados violentos -como es el caso de los E.P.requieren contar con un mayor contacto interrelacional y promoción de acciones de cuidado. En otras palabras, fortalecer una cultura del cuidado que favorezca la autoconsciencia y la búsqueda de respaldo, identificación y empatía con los compañeros de trabajo. Así, se considera que las reuniones para compartir los logros y dificultades laborales pueden ser espacios que podrían conseguir este objetivo.

Finalmente, lo anterior está estrechamente relacionado a la categoría de diálogo y coordinación entre áreas, donde se reconoce la importancia de la comunicación efectiva y asertiva al interior del espacio laboral, tanto para cumplir el objetivo institucional como para fortalecer el bienestar de los trabajadores. Como señalan Karl y Fisher (2013), los espacios grupales de discusión, donde se promueve la 
comunicación auténtica y efectiva dentro del espacio laboral, fomentan la retroalimentación cara a cara, brindan información e impulsan la colaboración entre trabajadores. Como consecuencia, tienen impacto en la disminución del SAP. No obstante, para que sean funcionales y productivos, los espacios de diálogo deben estar basados en la comunicación empática, donde se puedan expresar las emociones e ideas para reflexionar sobre las mismas.

En ese sentido, en el caso del sistema penitenciario, se debe empezar a analizar la importancia de la complementariedad entre las áreas; y la función que cada una de ellas cumple en la resocialización de las personas privadas de libertad, así como, en la gestión del sistema penitenciario. Es así que nuevamente se proponen espacios para propiciar la reflexión de las dificultades, en este caso no solamente para generar redes de soporte frente a los obstáculos y emociones que puedan aparecer por trabajar en el contexto penitenciario (como es el caso de la categoría integración entre áreas), sino en la implementación de esta estrategia caracterizada por analizar y esclarecer los objetivos penitenciarios a nivel institucional. Asimismo, estos espacios pueden focalizar las funciones de cada una de las áreas y estrategias de gestión laboral que impulsen acciones conjuntas entre ellas. Por consiguiente, implicaría construir una cultura institucional basada en la cooperación e intercambio.

\section{Fortalecimiento de habilidades personales y profesionales}

Los participantes también proponen que para eliminar el SAP, la institución debe enfocarse en los trabajadores, tanto de manera personal como profesional. Por tanto, a partir de la categoría habilidades personales, los participantes proponen que para enfrentar el estrés deben contar con actividades psicológicas y de desarrollo de capacidades de manera institucionalizada y sostenida en el tiempo. Impulsar este tipo de acciones coincide con lo propuesto por Karl y Fisher (2013), quienes reconocen la necesidad de la comunicación con uno mismo como estrategia de prevención del SAP. Los autores refieren que dentro del entorno laboral se debe organizar espacios donde se desarrolle la autoobservación de los síntomas como parte de una política de prevención del SAP. Así, este pedido puede comprenderse desde la necesidad de los trabajadores de contar con mejores herramientas personales que posibiliten la autoidentificación de síntomas, la autoregulación de conductas y la construcción de estrategias personales que ayuden a enfrentarlos (Karl, \& Fisher, 2013).

En la misma línea, proponen que debe impulsarse el fortalecimiento de habilidades profesionales que aporte a contar con mejores herramientas específicamente para el contexto penitenciario. Este pedido se alinea a la necesidad de sentirse seguros dentro de su espacio laboral, y buscar respuestas y soluciones concretas a la compleja realidad penitenciaria. Asimismo, el acceso a las capacitaciones profesionales, tal como señalan Ballard y Grawitch (2017), debe ser considerado justo y transparente. Es decir, los autores sostienen que la confianza y la justicia organizacional son factores centrales para establecer espacios psicológicamente saludables. Entonces, esta categoría no sólo implica brindar mayores herramientas al personal, sino que, también supone reflexionar y cambiar los procedimientos dentro de la gestión institucional, considerando criterios justos y transparentes.

La investigación da cuenta de diversas problemáticas y las estrategias que podrían ser impulsadas por la institución desde el punto de vista de sus trabajadores. Si bien el estudio se concentra en el contexto penitenciario peruano, esta mirada puede ampliarse para comprender la necesidad de implementar intervenciones sistémicas y complejas para enfrentar el SAP. Es decir, considerando programas elaborados desde una mirada multidimensional de la problemática y sus posibles estrategias de afrontamiento. Entonces, para lograr transformaciones estructurales que apunten a fortalecer el bienestar de los trabajadores, se deben proponer programas que busquen impactar a nivel institucional y organizacional, al fortalecimiento de habilidades personales y profesionales y a la construcción de relaciones interpersonales asertivas. Son dimensiones dinámicas, que dialogan entre si y que su transformación requiere de una comprensión sistémica que apunte a la construcción de espacios psicológicamente saludables.

Frente a ello, también se propone revisar el modelo de Maslach y Leiter (2014) para implementar programas para enfrentar el estrés laboral, quienes plantean la necesidad de impulsar acciones que respondan a problemáticas consideradas urgentes por los trabajadores, pero de manera colaborativa y sostenible. Asimismo, estos programas deben contar con estrategias diversas que respondan a la multicausalidad del SAP.

Asimismo, los programas deben organizarse pensando en el bienestar de los trabajadores, reconociendo las estrategias que vienen impulsando y sus opiniones sobre el entorno laboral (Demerouti, 2014). La posibilidad de accionar desde "el abajo hacia arriba" (Demerouti, 2014), coloca a los trabajadores en un rol activo que impulsa autonomía, motivación y compromiso (Ballard, \& Grawitch, 2017; Leiter, \& Maslach, 2014), posibilitando afianzar intervenciones a largo plazo (Walter, Plaumann, \& Krugman, 2013) que permitan transformaciones estructurales (Ballard, \& Grawitch, 2017; Demerouti, 2014). Como señalan Kelloway y Dimoff (2017), al ser necesidades 
y recomendaciones señaladas por los participantes, el reconocerlas e implementarlas puede ser el primer paso para construir programas donde se considere las opiniones y participación de los trabajadores.

Se sostiene que la eficacia de las estrategias depende de la formación de capacidades de los líderes, lo cual podría ser una línea de investigación para futuras propuestas. Siguiendo a Kelloway y Dimoff, (2017), los líderes juegan un rol central en la salud de los trabajadores en el espacio laboral. Son ellos, quienes a través de actitudes que promuevan el soporte social, la motivación y el compromiso, favorecen la satisfacción laboral, disminuyen el estrés y, por tanto, reducen la prevalencia de SAP.

\section{Referencias}

Ahola, K.; Toppinen-Tanner, S., \& Seppanen, J. (2017). Interventions to alleviate burnout symptoms and to support return to work among employees with burnout: Systematic review and meta-analysis. Burnout Research, 4 1-11.

http://dx.doi.org/10.1016/j.burn.2017.02.00 $12213-0586 /$

Aydemir, O., \& Icelli, I. (2013). Burnout: Risk factors. En: S. Bährer-Kohler, (Ed.), Burnout for experts: Prevention in the context of living and working (pp.119-143). New York, NY: Springer. http://dx.doi.org/10.1007/978-1-4614-4391$\underline{9}$

Ballard, D., \& Grawitch, M. (2017). Psychologically healthy workplace practices and employee well-being. En R. Burke y K. Page (Eds.). Research Handbook on Work and Well-Being (pp.494-509). Cheltenham: Edward Elgar Publishing Limited.

Beck, M., Bryman, A., \& Futing, L. (2004). The Sage encyclopedia of social science research methods. New Delhi, India: SAGE. http://dx.doi.org/10.4135/9781412950589

Bracco, L., Valdez, R., Wakeham, A., \& Velázquez, T. (En prensa). Síndrome de Agotamiento Profesional y trabajadores penitenciarios peruanos. Una mirada cualitativa a los factores institucionales y sociales. Revista Colombiana de Psicología.

Bracco, L., Wakeham, A., Valdez, R., \& Velázquez, T. (En prensa). Síndrome de Agotamiento Profesional, Variables Sociodemográficas y Condiciones Laborales en Trabajadores
Penitenciarios Peruanos. Apuntes en Psicología. Braun, V., \& Clarke, V. (2006). Using thematic analysis in psychology. Qualitative Research In Psychology, 3(2), 77-101. http://dx.doi.org/10.1191/1478088706qp063o a

Breevart, K; Bakker, A; Hetland, J., \& Hetland, H. (2014). The influence of constructive and destructive leadership behaviors on follower burnout. En: M. Leiter; A. Bakker, C. Maslach, (Eds.)., Burnout at work. a psychological perspective. London, UK: Psychology Press. Taylor and Francis Group.

Cáceres, C. (2013). Burnout y condiciones laborales en enfermeras y técnicas de cuidados intensivos neonatales (tesis de grado), Pontificia Universidad Católica del Perú, Lima, Perú.

Defensoría del Pueblo. (2013). Lineamientos para la implementación de las reglas de Bangkok en el Sistema Penitenciario Peruano. Lima, Perú: Programa de Asuntos Penales y Penitenciarios.

Defensoría del Pueblo. (2017). Quiénes somos: Nuestra misión. Disponible en https://www.defensoria.gob.pe/defensoria.ph $\mathrm{p}$

Demerouti, E. (2014). Individual strategies to prevent burnout. En: M. Leiter; A. Bakker, \& C. Maslach, (Eds.), Current issues in work and organizational psychology. Burnout at work. A psychological perspective (pp. 32-56). New York, NY: Psychology Press, Taylor and Francis Group.

Flick, U. (2018). Designing qualitative research. Los Angeles, CA: Sage.

García, J., \& Herrero, S. (2010). Revisión teórica del síndrome de burnout y su investigación en el contexto penitenciario. Apuntes de Psicología, 28(1), 83-107.

Gaudet, S., \& Robert, D. (2018). A journey through qualitative research: from designing to reporting. Los Angeles, CA: Sage.

Gil-Monte, P. (2005). El síndrome de quemarse por el trabajo (burnout): Una enfermedad laboral en la sociedad del bienestar. Madrid, España: Pirámide. http://dx.doi.org/10.13140/2.1.4614.8806

Gomes, R. (2012). Análisis e interpretación de datos de investigación cualitativa. En M. C. De Souza, S. Ferreira, \& R. Gomes, (Eds.), Investigación social. Teoría, método y creatividad (pp. 607- 
114). Buenos Aires, Argentina: Lugar Editorial. Hemmeter, U. (2013). Treatment of burnout. Overlap of diagnosis. En: S. Bahrer-Kohler (Ed.). Burnout for experts. Prevention in the context of living and working (pp. 73-87). New York, NY: Springer.

Hennink, M.; Hutter, I., \& Bailey, A. (2011). Qualitative research methods. London: Sage.

Hernández, R; Fernández, C., \& Baptista, P. (2010). Metodología de la investigación. México, D. F.: Mc Graw-Hill Interamericana.

Hernández, L., Fernández, B., Ramos, F., \& Contador, I. (2006). El síndrome de burnout en funcionarios de vigilancia de un centro penitenciario. International Journal of Clinical and Health Psychology, 6(3), 599-611.

Hurrell, J. (2005). Organizational stress interventions'. En: J. Barling; K. Kelloway, \& M. Frone, (Eds.), Handbook of work stress (pp. 623-646). Thousand Oaks, CA: Sage.

Instituto Nacional Penitenciario. (2016). Reporte de cumplimiento misional del Instituto Nacional Penitenciario y estado situacional de los sistemas administrativos. Anexo $N^{\circ} 2$. Periodo: agosto 2011-julio 2016. Disponible en https://www.minjus.gob.pe/wpcontent/uploads/2016/08/INFORME-DETRANSFERENCIA-DE-GESTION-INPE.pdf

Instituto Nacional Penitenciario. (INPE). (2018). Informe estadístico penitenciario 2018. Disponible en

https://www.inpe.gob.pe/normatividad/estad\%C3 \%ADstica/1225-informe-julio-2018/file.html

Karl, D., \& Fischer, M. (2013.). Prevention and communication: A most effective tailored treatment strategies for burnout. En: S. Bahrer-Kohler, (Ed.), Burnout for experts. Prevention in the context of living and working (pp. 185-200). New York, NY: Springer.

Kelloway, E. y Dimoff, J. (2017). Leadership interventions to improve well-being. En: R. Burke, \& K. Page, (Eds.), Research handbook on work and well-being (pp. 435-452). Cheltenham, UK: Edward Elgar Publishing.

Llaja, V., Sarriá, J., \& García, P. (2007). Inventario "Burnout" de Maslach \& Jackson - muestra peruana. Lima, Perú: Centro Interdisciplinario en Neuropsicología.

Leiter, M., \& Maslach, C. (2014). Interventions to prevent and alleviate burnout. En: M. Leiter,
A. Bakker, \& C. Maslach, (Eds.), Current issues in work and organizational psychology: Burnout at work. A psychological perspective (pp. 145167). New York: Psychology Press, Taylor and Francis Group.

Maslach, C. (1993). Burnout: A multidimensional perspective. En W. B. Schaufeli, C. Maslach, \& T. Marek (Eds.), Series in applied psychology: Social issues and questions. Professional burnout: Recent developments in theory and research (pp. 19-32). Philadelphia, PA: Taylor \& Francis.

Maslach, C. (2003). Burnout: Cost of caring. Cambridge, UK: Malor Books.

Maslach, C., Schaufeli, W., \& Leiter, M. (2001). Job burnout. Annual Review of Psychology, 52, 397.

McCormack, N., \& Cotter, C. (2013). Managing burnout in the workplace. A guide for information professionals. Oxford: Chandos Publishing. https://doi.org/10.1146/annurev.psych.52.1.3 $\underline{97}$

Mercado-Salgado, P., \& Gil-Monte, P. (2010). Influencia del compromiso organizacional en la relación entre conflictos interpersonales y el síndrome de quemarse por el trabajo (burnout) en profesionales de servicio (salud y educación). Innovar: Revista de Ciencias Administrativas y Sociales, 20(38), 161-174.

Ministerio de Justicia y Derechos Humanos (2016). ¿Qué tanto saber del Sistema Penitenciario en el Perú? Lima, Perú: Observatorio Nacional de Política Criminal.

Muñoz, J. (2003). Manual de Análisis cualitativo de datos textuales con ATLAS.ti (2.4). Disponible en

http://www.ugr.es/ textinfor/documentos/m anualatlas.pdf

Navarro, I., López, B., Heliz, J., \& Real, M. (2018). Estrés laboral, burnout y estrategias de afrontamiento en trabajadores que intervienen con menores en riesgo de exclusión social. Aposta. Revista de Ciencias Sociales, 78, 68-96.

Olabarria, B., \& Mansilla, F. (2007). Ante el burnout: Cuidados a los equipos de salud mental. Revista de Psicopatología y Psicología Clínica, 12(1), 114.

Osorio, J., \& Cárdenas, L. (2017). Estrés laboral: estudio de revisión. Revista DiversitasPerspectivas en Psicología, 13(1), 81-90. 
Rodríguez, R. (2014). Síndrome de burnout en guardianes penitenciarios. Revista Colombiana de Psiquiatría, 43(3), 146-153. https://doi.org/10.1016/j.rcp.2014.03.001

Rodríguez, F.; Rubin, A.; Bringas, C.; Álvarez, E., \& Herrero, J. (2015). Efecto del clima emocional en el desarrollo del estrés laboral del funcionario penitenciario: un estudio predictivo. Ansiedad y Estrés, 21(2-3), 195205.

Santivañez, A. (2016). Burnout y salud en profesionales de enfermería de un hospital nacional de Huancayo. Tesis para optar por el título de Licenciada en Psicología. Lima, Perú: Pontificia Universidad Católica del Perú.

Schaufeli, W., Maslach, C., \& Marek, T. (1993). Professional burnout: Recent developments in theory and research. Londres, UK: Taylor \&
Francis.

Velázquez, T.; Bracco, L.; Hildendrand, A.; Wakeham, A.; Valdez, R.; Florentini, M.; ..., \& Oviedo, V. (2015). Síndrome de agotamiento profesional en trabajadores de tratamiento de dos establecimientos penitenciarios de Lima. Apuntes de Psicología, 33(2), 57-65.

Walter, U.; Plaumann, M., \& Krugmann, C. (2013). Burnout intervention. En S. Bahrer-Kohler, (Ed.), Burnout for experts. Prevention in the context of living and working (pp. 223-246). New York, NY: Springer.

Yepez, C. (2015). Percepción del trabajo en la comunidad en miembros de equipos de una institución de salud mental. Tesis para optar por el título de Licenciada en Psicología. Lima: Pontificia Universidad Católica del Perú. 